\title{
ANALYSIS OF THE INFLUENCE OF THE NEIGHBOURING ELEMENTS IN AN HGMS MATRIX ON THE MAGNETIC FIELD DISTRIBUTION IN THE VICINITY OF ONE ELEMENT
}

\author{
Yu.S. MOSTYKA*, V.Yu. SHUTOV and L.Z. GREBENYUK \\ The National Mining University, 19 Karl Marks Prospect, \\ 49027 Dnepropetrovsk, The Ukraine
}

(Received 27 April 2004; In final form 14 May 2004)

\begin{abstract}
This article theoretically researches the magnetic field distribution within HGMS matrix free space - a stack of orderly placed cylinder bars. It is shown that, in near vicinity of any matrix bar, the distribution of the magnetic field practically coincides with the field distribution around a single cylinder. Relative differences of force distribution characteristic $f(x, y)=H|\operatorname{grad} H|$ are calculated for the bar stack and single cylinders depending upon the relative distance between a given field point and the axis of the bar.
\end{abstract}

Keywords: Magnetic separation; Matrix; Magnetic field; Magnetic force

\section{INTRODUCTION}

It is possible to use stacks of orderly placed bars as an HGMS matrix [1,2]. Highgradient magnet separators, made in the Czech Republic, are currently used Ukrainian installations that had treated manganese ores. Their matrices are the grids of bars (or rods); that is, the stacks of orderly placed cylindrical ferromagnetic elements.

In order to predict separation characteristics it is necessary to calculate the distribution of the magnetic field in a working zone under given values of magnetic induction and the geometric parameters of the bar grids. Calculation of the magnetic field in the space between the bars can be performed by means of numerical solutions of magnetic field equations under given boundary conditions. In some cases, however, those calculations can be replaced by easier calculations according to

*Corresponding author. E-mail: mostyka@ukr.net 
analytical formulae. In particular, such a simplification is relatively practicable for the small area in the neighborhood of each matrix element.

Previous calculations show that if one is limited to the vicinity of a cylindrical bar, such as $r<(0.2-0.3) L$ ( $r$ is distance from matrix cylinder element axis to a given point; $L$ is distance between the axes of two contiguous bars), the distribution of magnetic field strength $H(x, y)$ of bars system can be approximately replaced by the distribution of a round single cylinder. In a case similar to this, the $H(x, y)$ distribution is calculated in accordance with well-known analytic formulae [1].

\section{THEORETICAL MODEL}

Let us consider a magnetic separator matrix made up of a grid of cylindrical elements which are parallel to each other and the centres of their cross-sections in the $(x, y)$ plane normal for cylinder axes are situated in square net points of which the cell dimension is $L \times L$. Such a matrix scheme was considered in [3].

Calculations show that while researching the parameters of the magnetic field existent in the free space of such a matrix, it is possible to examine a system consisting of nine cylinder elements as outlined in Fig. 1. The matrix is in external uniform field whose strength is $H_{0}$ (and magnetic induction $B_{0}$ ).

If the distance $L$ is much shorter than the length of the cylindrical element, the magnetic field can be supposed to be planary parallel so that the parameters of magnetic field are functions of two $(x, y)$ space coordinates only.

It is obvious that the degree of influence of the neighbouring elements on the magnetic field parameters near one of the elements to be examined (element 1 , Fig. 1) depends upon a geometric parameter $L / d_{w}$, where $d_{w}$ is a diameter of the cylinder element.

One can assume that this degree of influence also depends upon the magnetic induction $B_{0}$ of the external magnetic field, saturation induction $B_{s}$ of ferromagnetic elements, and on other parameters. The analysis of the influence of the two aforementioned factors $\left(L / d_{w}\right.$ and $\left.B_{0}\right)$ is given later.

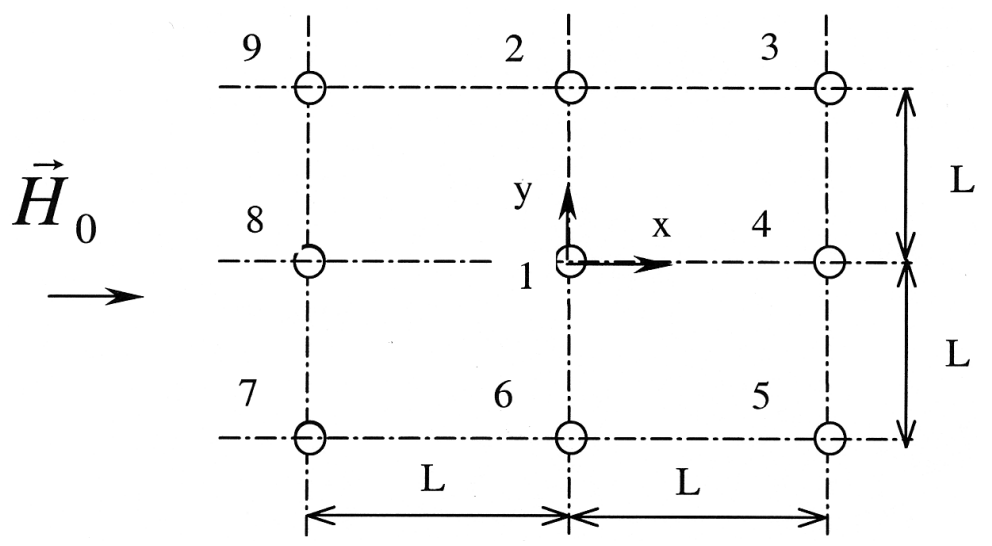

FIGURE 1 Schematic diagram of location of ferromagnetic matrix elements. 
Let us employ the formulae of magnetic field strength and force characteristic $f$ for the system including many cylinder elements [3]:

$$
\begin{aligned}
f & =\left(f_{x, \Sigma}^{2}+f_{y, \Sigma}^{2}\right)^{1 / 2}, \\
f_{x, \Sigma} & =H_{x, \Sigma} \frac{\partial H_{x, \Sigma}}{\partial x}+H_{y, \Sigma} \frac{\partial H_{y, \Sigma}}{\partial x} ; \\
f_{y, \Sigma} & =H_{x, \Sigma} \frac{\partial H_{x, \Sigma}}{\partial y}+H_{y, \Sigma} \frac{\partial H_{y, \Sigma}}{\partial y} ; \\
H_{x, \Sigma} & =H_{0}+\sum_{i=1}^{n} H_{x, i} ; \\
H_{y, \Sigma} & =\sum_{i=1}^{n} H_{y, i} ; \\
H_{x, i} & =H_{0} A r_{w}^{2} \frac{x_{i}^{2}-y_{i}^{2}}{\left(x_{i}^{2}+y_{i}^{2}\right)^{2}} \\
H_{y, i} & =H_{0} A r_{w}^{2} \frac{2 x_{i} y_{i}}{\left(x_{i}^{2}+y_{i}^{2}\right)^{2}},
\end{aligned}
$$

where $x_{i}, y_{i}$ are the coordinates of random field point to be determined along the $(x, y)$ axes relative to the centre of the $i$-element cross-section; $r_{w}=d_{w} / 2$ and $A=\mu_{w}-\mu_{f} / \mu_{w}+\mu_{f}$, where $\mu_{w}$ and $\mu_{w}$ are the magnetic permeabilities of the wire material and fluid medium, respectively.

It is supposed here that the strength of the external field imposed on each element of the rod grid is still equal to $H_{0}$ as in the case of a single cylinder. Such a simplification is acceptable if the distance between axes of the rods is relatively large, namely $L / d_{w} \geq 4$.

When $i=1$ (for the centre of nine elements system), the coordinates $\left(x_{i}, y_{i}\right)$ are equal to the $(x, y)$ coordinates: $x_{1}=x ; y_{1}=y$.

For other values of $i$ index the coordinates $\left(x_{i}, y_{i}\right)$ are defined by the formulae:

$$
\begin{aligned}
& x_{2}=x_{6}=x ; \quad x_{3}=x_{4}=x_{5}=x-L ; \quad x_{1}=x_{8}=x_{9}=x+L \\
& y_{2}=y_{3}=y_{9}=y-L ; \quad y_{4}=y_{8}=y ; \quad y_{5}=y_{6}=y_{7}=y+L
\end{aligned}
$$

We shall define a relative error as:

$$
\Delta f=\frac{f_{1}-f}{f}=\frac{f_{1}}{f}-1,
$$

where $f$ and $f_{1}$ are the values of $H|\operatorname{grad} H|$ force parameter calculated in the given field point according to a system that includes nine cylindrical elements and a single element, respectively.

In addition to the orthogonal coordinate system let us introduce the polar coordinate system $(r, \theta)$ whose centre coincides with the centre of the cross-section of the first element (to be central in the system of nine elements); the $\theta$ angle is measured from the $x$ axis. 
The maximum error values $\Delta f$ on the $f=$ const isolines will be obtained if $\theta=0$. So let us consider error $\Delta f$ as it depends upon the distance to the centre of an element specifically for $\theta=0$.

In this case $y=0$; that is the point $f$ value thus determined lies on the magnetic field symmetry axis. Therefore, from Eq. (3), and taking into account Eqs. (4)-(7) and the link of coordinates given by Eqs. (8) and (9), it is possible to obtain:

$$
\left(f_{y, \Sigma}\right)_{\theta=0}=0 .
$$

From Eqs. (1) and (2) we have:

$$
(f)_{\theta, 0}=\left(f_{x, \Sigma}\right)_{\theta, 0}=\left[H_{x, \Sigma} \frac{\partial H_{x, \Sigma}}{\partial x}+H_{y, \Sigma} \frac{\partial H_{y, \Sigma}}{\partial x}\right]_{\theta=0}
$$

For $\theta=0, y_{1}=y_{4}=y_{8}=0 ; y_{2}=y_{3}=y_{9}=-L ; y_{5}=y_{6}=y_{7}=L$, we obtain, from Eq. (7):

$$
\left(H_{y, i}\right)_{\theta=0}=0 \quad \text { when } \quad i=1,4 \text { and } 8
$$

where $i=2$ and 6 for any point at $x$ axis we get:

$$
\left(H_{y, 2}\right)_{\theta=0}=-\left(H_{y, 6}\right)_{\theta=0}
$$

Analogous relationship takes place when $i=3$ and 5 , and when $i=7$ and 9:

$$
\left(H_{y, 3}\right)_{\theta=0}=-\left(H_{y, 5}\right)_{\theta=0} ; \quad\left(H_{y, 7}\right)_{\theta=0}=-\left(H_{y, 9}\right)_{\theta=0}
$$

Taking into account Eqs. (11)-(13), and from Eq. (5) for $n=9$ we get:

$$
\left(H_{y, \Sigma}\right)_{\theta=0}=0
$$

Substituting Eqs. (4) and (14) into Eq. (10) for $n=9$ we have:

$$
(f)_{\theta=0}=\left[\left(H_{0}+\sum_{i=1}^{9} H_{x, i}\right) \sum_{i=1}^{9} \frac{\partial H_{x, i}}{\partial x}\right]_{\theta=0}
$$

then taking into consideration Eq. (6) we can obtain:

$$
(f)_{\theta=0}=2 A H_{0}^{2}\left[\left(1+A r_{w}^{2} \sum_{i=1}^{9} \frac{x_{i}^{2}-y_{i}^{2}}{r_{i}^{4}}\right) r_{w}^{2} \sum_{i=1}^{9} \frac{x_{i}\left(3 y_{i}^{2}-x_{i}^{2}\right)}{r_{i}^{6}}\right]_{\theta=0}
$$

or

$$
(f)_{\theta=0}=2 A \frac{H_{0}^{2}}{r_{w}}\left[\left(1+A \sum_{i=1}^{9} \frac{\tilde{x}_{i}^{2}-\tilde{y}_{i}^{2}}{\tilde{r}_{i}^{4}}\right) \sum_{i=1}^{9} \frac{\tilde{x}_{i}\left(3 \tilde{y}_{i}^{2}-\tilde{x}_{i}^{2}\right)}{\tilde{r}_{i}^{6}}\right]_{\theta=0}
$$


where $r_{i}=\sqrt{x_{i}^{2}+y_{i}^{2}}$;

$$
\tilde{x}_{i}=x_{i} / r_{w} ; \quad \tilde{y}_{i}=y_{i} / r_{w} ; \quad \tilde{r}_{i}=r_{i} / r_{w}
$$

Introducing designation:

$$
Q_{i}=\frac{\tilde{x}_{i}^{2}-\tilde{y}_{i}^{2}}{\tilde{r}_{i}^{4}} ; \quad S_{i}=\frac{\tilde{x}_{i}\left(3 \tilde{y}_{i}^{2}-\tilde{x}_{i}^{2}\right)}{\tilde{r}_{i}^{6}}
$$

we have:

$$
(f)_{\theta=0}=2 A \frac{H_{0}^{2}}{r_{w}}\left[\left(1+A \sum_{i=1}^{9} Q_{i}\right) \sum_{i=1}^{9} S_{i}\right]_{\theta=0}
$$

Taking into account $x_{1}=x, y_{1}=y$ and Eqs. (8) and (9), it is possible to obtain the following expressions for $Q_{i}, S_{i}(i=1,2, \ldots, 9)$ :

$Q_{1}=\frac{1}{\tilde{x}^{2}} ; \quad S_{1}=-\frac{1}{\tilde{x}^{3}}$

$Q_{2}=\frac{\tilde{x}^{2}-\tilde{L}^{2}}{\left(\tilde{x}^{2}+\tilde{L}^{2}\right)^{2}} ; \quad S_{2}=\frac{\tilde{x}\left(3 \tilde{L}^{2}-\tilde{x}^{2}\right)}{\left(\tilde{x}^{2}+\tilde{L}^{2}\right)^{3}}$

$Q_{3}=\frac{\tilde{x}^{2}-2 \tilde{x} \tilde{L}}{\left(\tilde{x}^{2}-2 \tilde{x} \tilde{L}+2 \tilde{L}^{2}\right)^{2}} ; \quad S_{3}=\frac{-\tilde{x}^{3}+3 \tilde{x}^{2} \tilde{L}-2 \tilde{L}^{3}}{\left(\tilde{x}^{2}-2 \tilde{x} \tilde{L}+2 \tilde{L}^{2}\right)^{3}}$

$Q_{4}=\frac{1}{(\tilde{x}-\tilde{L})^{2}} ; \quad S_{4}=-\frac{1}{(\tilde{x}-\tilde{L})^{3}}$

$Q_{5}=Q_{3} ; \quad S_{5}=S_{3}$ (since $x_{3}=x_{5} ; y_{3}=-y_{5}$ and $y_{i}^{2}$ is in $Q_{i}$ and $S_{i}$ formulae)

$\mathrm{Q}_{6}=Q_{2} ; S_{6}\left(\right.$ since $x_{2}=x_{6} ; y_{2}=-y_{6}$ and $\left.y_{2}^{2}=y_{6}^{2}\right)$

$Q_{7}=\frac{\tilde{x}^{2}+2 \tilde{x} \tilde{L}}{\left(\tilde{x}^{2}+2 \tilde{x} \tilde{L}+2 \tilde{L}^{2}\right)^{2}} ; \quad S_{7}=\frac{-\tilde{x}^{3}-3 \tilde{x}^{2} \tilde{L}+2 \tilde{L}^{3}}{\left(\tilde{x}^{2}+2 \tilde{x} \tilde{L}+2 \tilde{L}^{2}\right)^{3}} ;$

$Q_{8}=\frac{1}{(\tilde{x}+\tilde{L})^{2}} ; \quad S_{8}=-\frac{1}{(\tilde{x}+\tilde{L})^{3}}$

$Q_{9}=Q_{7} ; \quad S_{9}=S_{7}\left(\right.$ since $x_{7}=x_{9} ; y_{7}=-y_{9}$ and $\left.y_{7}^{2}=y_{9}^{2}\right)$

where $\tilde{L}=L / r_{w}$.

The formulae (15) and (16) are an analytical expression for the force parameter $f=H|\operatorname{grad} H|$ for a nine cylinder element system in any point of those lying on the $x$ axis $(\theta=0)$.

For a single cylinder element we have:

$$
\left(f_{1}\right)_{\theta=0}=2 A \frac{H_{0}^{2}}{r_{w}}\left(1+\frac{A}{\tilde{x}^{2}}\right)\left(-\frac{1}{\tilde{x}^{3}}\right)
$$




\section{RESULTS}

Let us consider a distribution of the magnetic field force parameter $f=H|\operatorname{grad} H|$ in the $(x, y)$ plane, normal to the axis of the cylindrical element. Distribution variation $f(x, y)$ due to the variation of the magnetic induction $B_{0}$ under fixed value $L / d_{w}=4$ value can be seen in Figs. 2(a) and 2(b), where the results of calculations are shown as the lines of equal levels (isolines) of the $f$ parameter, in accordance with the $B_{0}$ values equal to 0.5 and $9 \mathrm{~T}$. Let us note that $f=$ const isolines are calculated close to the element 1 , which is at the centre of the nine cylinder elements in Fig. 1.

Absolute values of $f$ on the isolines were calculated for the case of $d_{w}=2 \mathrm{~mm}$. A procedure of calculating $f$ for other values of $d_{w}$ is described in [3]. As can be seen, $f(x, y)$ distributions for the values $B_{0}=0.5$ and $9 \mathrm{~T}$ are qualitatively similar and differ mainly
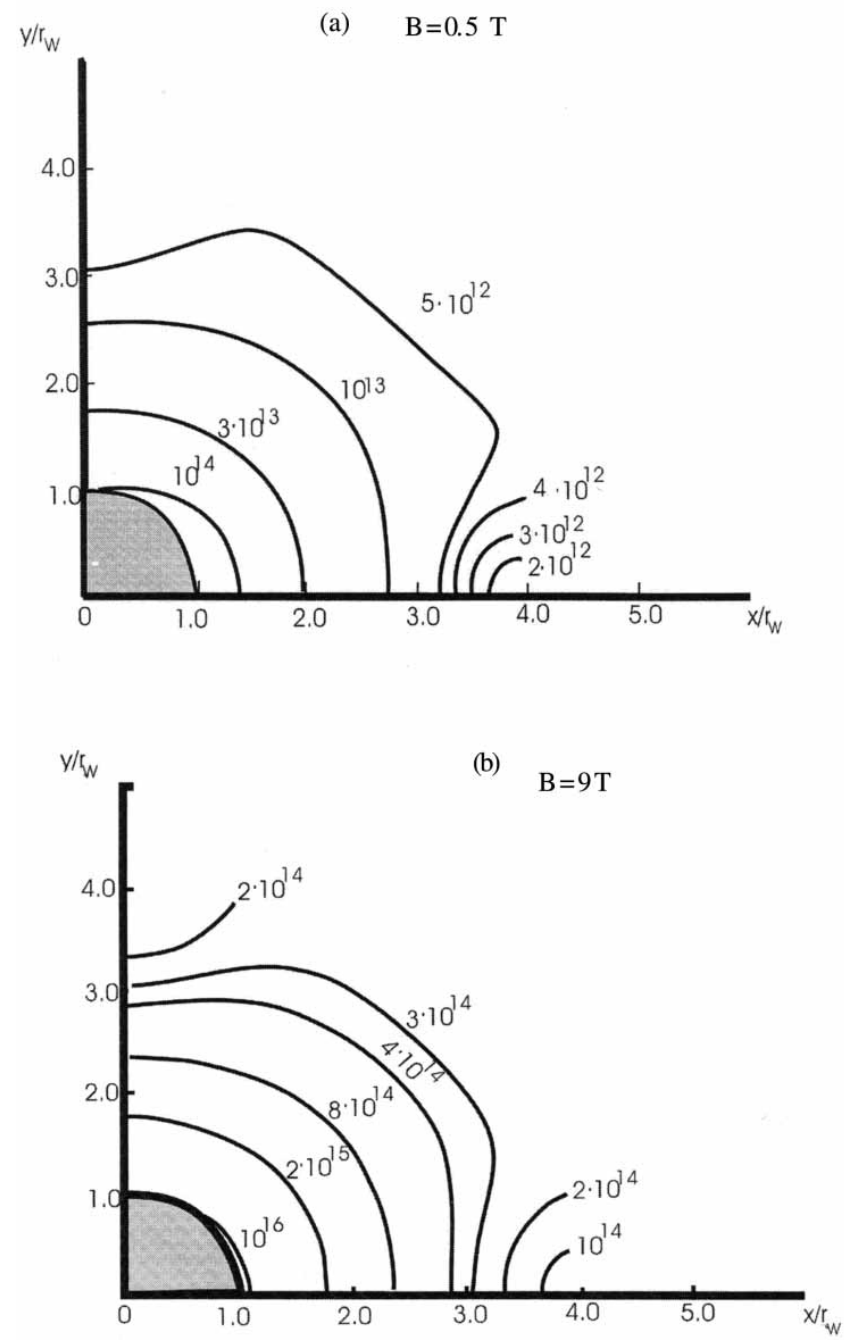

FIGURE 2 Isolines of $f=H|\operatorname{grad} H|\left(\mathrm{A}^{2} / \mathrm{m}^{3}\right)$ near cylindrical ferromagnetic element $\left(N=9, L / d_{w}=4\right.$, $d_{w}=2 \mathrm{~mm}$ ). 
in absolute values of $f$ on isolines occupying the same (or close) positions in the $(x, y)$ plane. It can also be seen that the $f(x, y)$ distributions have local peculiarities near the mean points, i.e. points at the centre of each line segment of length $L$ connecting the centres of the neighbouring elements. Those line segments are parallel to $x$ or $y$ axes. In Figs. 2(a) and 2(b), the coordinates of the mean points are $\tilde{x}=4$ if $\tilde{y}=0$ and $\tilde{x}=0$ if $\tilde{y}=4$, where $\tilde{x}=x / r_{w} ; \tilde{y}=y / r_{w} ; r_{w}=d_{w} / 2$.

The variations of the $f(x, y)$ distribution with the change of the relative distance $L / d_{w}$ between the axis of neighbouring cylindrical elements, under fixed value of $B_{0}=2 \mathrm{~T}$, are shown in Fig. 3(a)-(d), as $f=$ const isolines calculated close to the central element of nine cylinder elements; $L / d_{w}=4 ; 5 ; 6$ and 8 .

Comparing $f=$ const equal level line configuration under equal $f$ values for different $L / d_{w}$ one can conclude that in a near vicinity of the cylinder, distribution $f(x, y)$ practically does not depend upon $L / d_{w}$ but the distribution difference increases gradually as the given point approaches the middle point.

For example, for $L / d_{w}=4.0$ and 5.0 (Figs. 3(a) and 3(b)), $f=$ const isolines practically coincide for $f=8 \times 10^{13} ; 2 \times 10^{14} ; 8 \times 10^{14}$ and $2 \times 10^{15} \mathrm{~A}^{2} / \mathrm{m}^{3}$, while for $f=4 \times 10^{13} \mathrm{~A}^{2} / \mathrm{m}^{3}$ they are essentially different, and for $f=3 \times 10^{13} \mathrm{~A}^{2} / \mathrm{m}^{3}$ the isolines for $L / d_{w}=4.0$ and 5.0 differ even qualitatively: if $L / d_{w}=4,0$ the $f=3 \times 10^{13} \mathrm{~A}^{2} / \mathrm{m}^{3}$

(a)

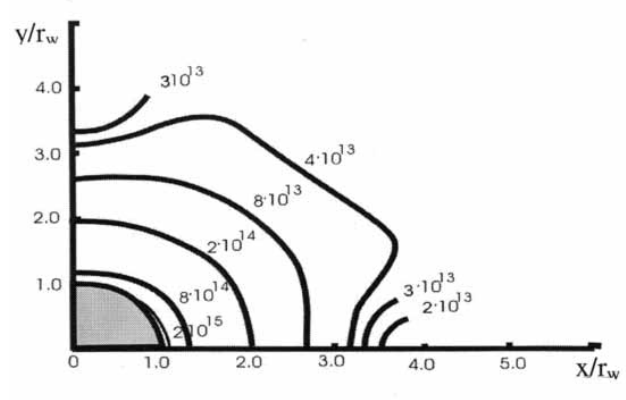

(c)

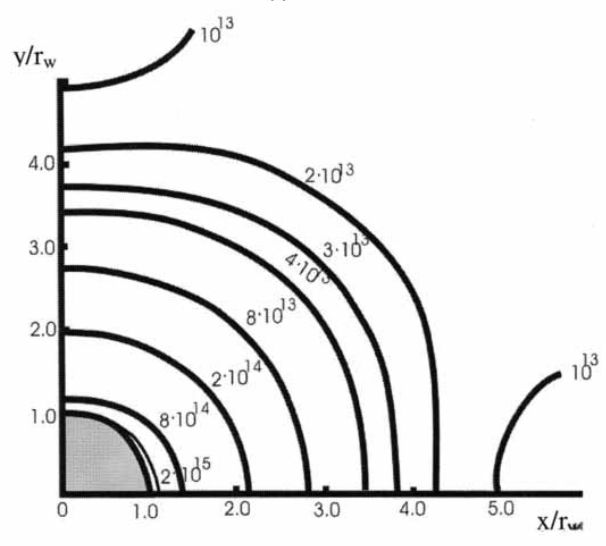

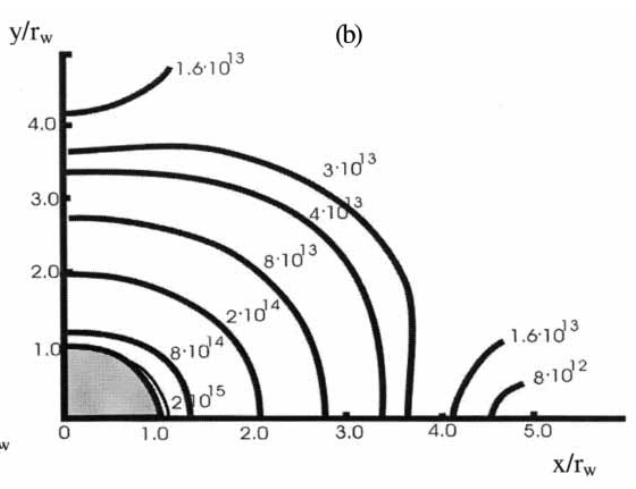

(d)

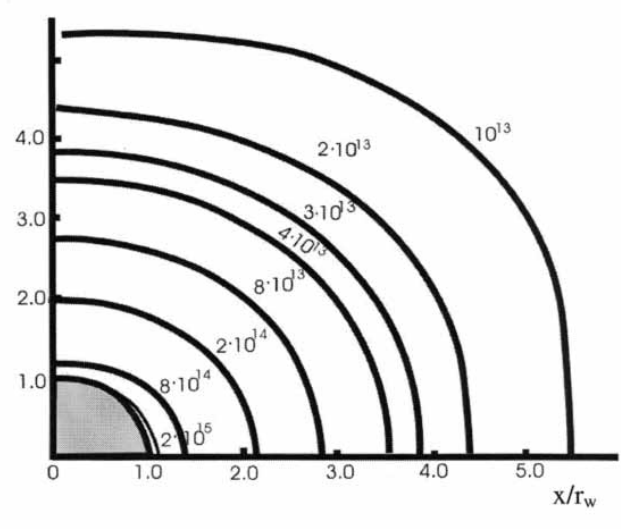

FIGURE 3 Isolines of $f=H|\operatorname{grad} H|\left(\mathrm{A}^{2} / \mathrm{m}^{3}\right)$ near a cylindrical ferromagnetic element $\left(N=9, B_{0}=2 \mathrm{~T}\right.$, $d 3_{w}=2 \mathrm{~mm}$ ): (a) $L / d_{w}=4$; (b) $L / d_{w}=5$; (c) $L / d_{w}=6$; (d) $L / d_{w}=8$. 
isoline encloses the vicinity of the middle point. When $L / d_{w}=5.0$, the cylindrical element is enclosed.

Consequently, as can be seen in Figs. 3(c) and 3(d), for $L / d_{w}=6.0$, the $f=10^{13} \mathrm{~A}^{2} / \mathrm{m}^{3}$ isoline encloses the vicinity of the mean point, and the isoline $f=2 \times 10^{13} \mathrm{~A}^{2} / \mathrm{m}^{3}$ encloses the cylindrical element. However, for $L / d_{w}=8.0$ both isolines enclose the cylindrical element.

Let us consider the influence of the matrix contiguous elements according to additional criterion, namely, the relative difference of the characteristic parameter $f(x, y)$ for a single cylinder element and for a system consisting of many such elements. This difference can be considered also as the calculation error of the force parameter $f$ according to the simplified model of a single cylinder element, in comparison with the calculation results on the basis of a more complex model of many elements.

The calculations indicate that the peak value of the error is achieved on a straight line passing through the centre of the cross-section of this cylinder parallel to the $H_{0}$ vector. If we examine the centre element belonging to the system of nine cylinder elements (Fig. 1) we shall see that the straight line coincides with the $x$ axis.

Using Eqs. (15)-(17) we can find the error $(f)_{\theta=0}=\left(f_{1} / f\right)_{\theta=0}-1$ at the points on the $x(\theta=0)$ axis for different $B_{0}$ and $L / d_{w}$ values. As it was pointed out earlier, the errors $f$ on the $f=$ const isolines achieve the maximum for $\theta=0$.

Figure 4 shows the $f=$ const isolines calculated according to formulae both for a single cylinder element and a system consisting of nine such elements (arranged according to the diagram in Fig. 1), for $L / d_{w}=4 ; B_{0}=0.5 \mathrm{~T}$. The $f_{1}=$ const isolines cross the $x$ axis at $\tilde{x}=2.0$ and $3.0\left(\tilde{x}=x / r_{w}\right)$ points. The analogous curves were plotted for $L / d_{w}=5$ and 6 for $B_{0}=0.2 ; 0.5 ; 1.0 ; 2 ; 4$ and $9 \mathrm{~T}$.

In addition, the values of the distance $r_{0}$ from cylinder axis to $f_{1}=$ const isoline intersection point with $x$ axis $(\theta=0)$ were varied in the range from $1.5 r_{w}$ to $0.45-L$.

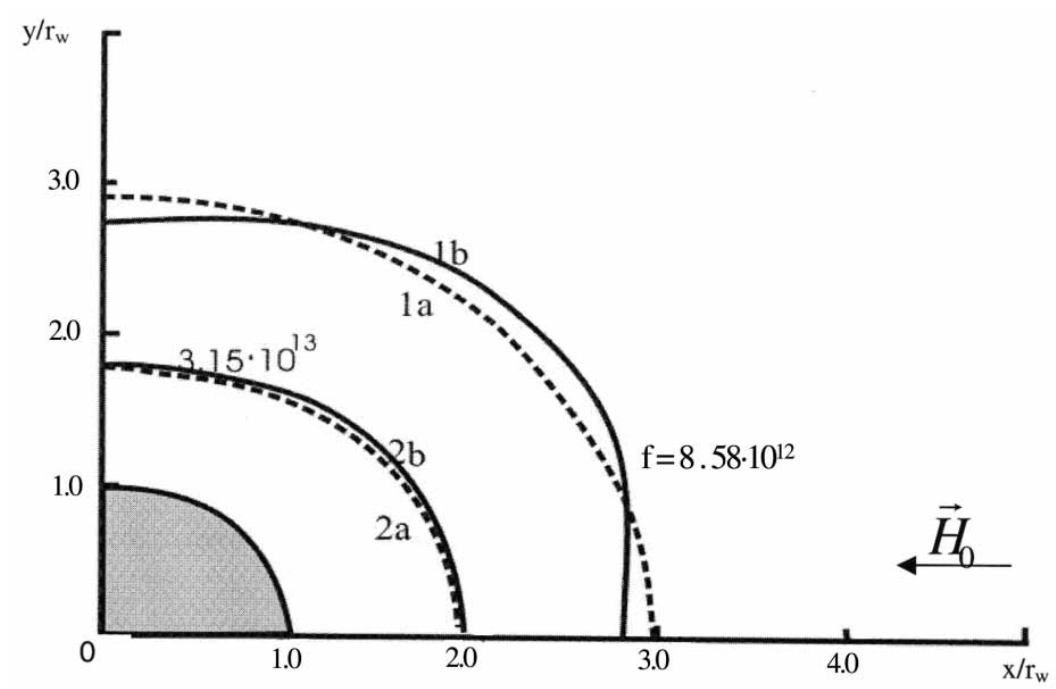

FIGURE 4 Isolines of $f=H|\operatorname{grad} \mathrm{H}|$ near a cylindrical ferromagnetic element $\left(B_{0}=0.5 \mathrm{~T}, L / d_{w}=4\right.$, $d_{w}=2 \mathrm{~mm}$ ) 1a, 2a-a single cylindrical ferromagnetic element; 1b, 2b-system of 9 cylindrical ferromagnetic elements. 


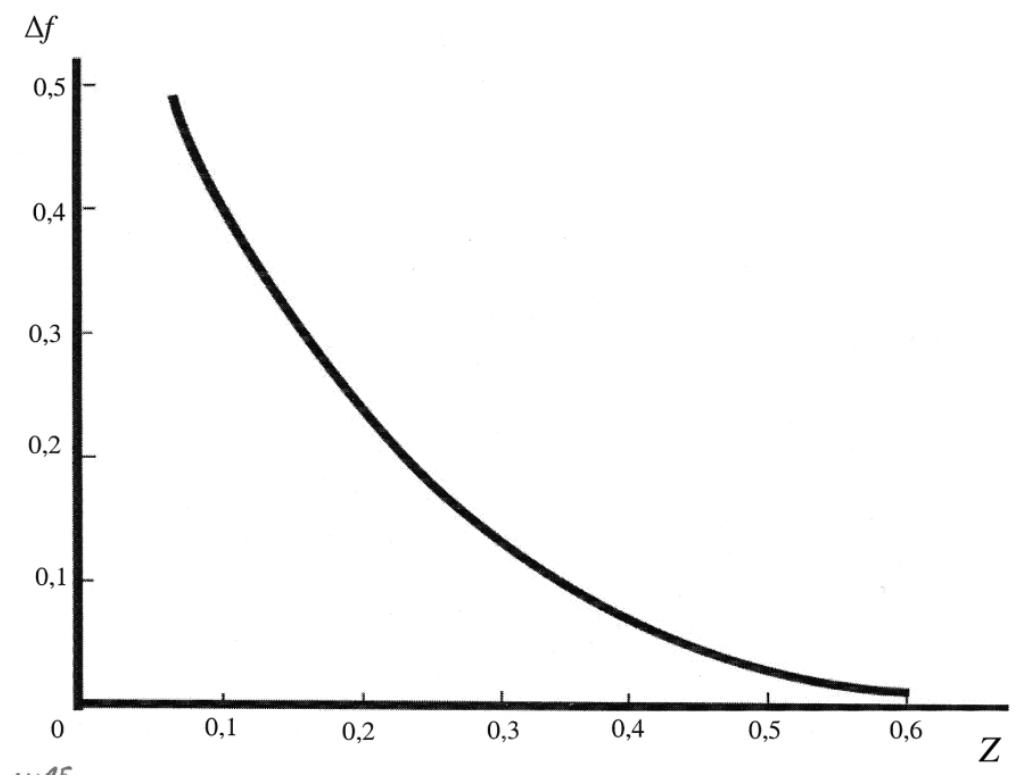

FIGURE 5 The dependence of the relative difference $\Delta f=\left(f_{1}-f\right) / f$ upon the relative distance $Z=\left(L / 2-r_{0}\right) /(L / 2)$.

The errors $\Delta f$ were expressed as dependence on a parameter $Z=\left(L / 2-r_{0}\right) /$ $(L / 2)=1-2 r_{0} / L$. This dependence, for $B_{0}=1 \mathrm{~T}$ and $L / d_{w}=4$ is shown in Fig. 5 . The dependence for other values of $B_{0}$, in the range from 0.2 to $9 \mathrm{~T}$, for $L / d_{w}=4$; 5 and 6 do not practically differ from the dependence shown in Fig. 5.

\section{CONCLUSION}

A theoretical investigation concerning the influence of the neighbouring elements in the ferromagnetic matrix on magnetic force parameter $f=H|\operatorname{grad} H|$, in the vicinity of one matrix element was carried out. In order to achieve this, relative differences $\Delta f$ of the $f$ value for a single cylinder element and for a system consisting of many such elements were calculated under different values of the magnet induction, relative distance $L / d_{w}$ between axes of cylinders and under different positions of field point at which the $f$ characteristic was being defined. It has been found that the $\Delta f$ dependence on $B_{0}$ was weak. The dependence of $\Delta f$ on the location of the field point can be shown as universal (from the point of view of different $L / d_{w}$ applicability) function of the geometric parameter $Z$. This parameter is the distance from a central point to a given field point expressed as a fractions of the $L / 2$ distance. The influence of contiguous elements qualitatively expressed by the value of $\Delta f$ is obtained, while approaching to a middle point. In the spherical area adjacent to the cylindrical element (diameter of the area smaller than $0.25 L$ ) the value of $\Delta f$ usually does not exceed $5 \%$, so the influence of neighbour elements can be neglected. 


\section{References}

[1] J. Svoboda, Magnetic Methods for the Treatment of Minerals, Elsevier, Amsterdam, 1987.

[2] N. Rezlescu, V. Badescu, E.B. Bradu and Gh. Iacob, The Principles of Magnetic Separation of Materials, Editura Academici Române, Bucharest, [in Romanian].

[3] V.I. Karmazin, Yu.S. Mostyka, V.Yu. Shutov and L.Z. Grebenyuk, The Research of HGMS Matrix Saturation Influence on Magnetic Field Force Characteristics, //Mining Informational and Analytical Bulletin. No. 3, Moscow Mining State University, 1998.

\section{AUTHORS BIOGRAPHIES}

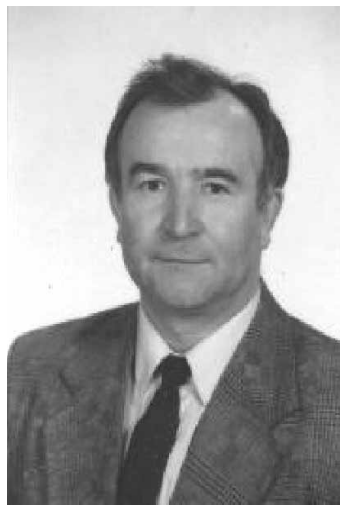

Mostyka, Youry graduated from Dnepropetrovsky Mining Institute in 1977 and acquired a profession of mining engineerelectrical. In 1986 he obtained his academic degree of Candidate of Technical Science (Ph.D.) in minerals beneficiation. The fields of his scientific interests include magnetic properties of minerals, theoretical and experimental research in separation of weakly-magnetic ores, ore beneficiation in strong magnetic fields and kinetics of high-gradient magnetic separation. Dr. Mostyka's practical work is concerned with the design of technology and equipment for beneficiation of ilmenite, rutile, zirconium ores, feldspar and garnet.

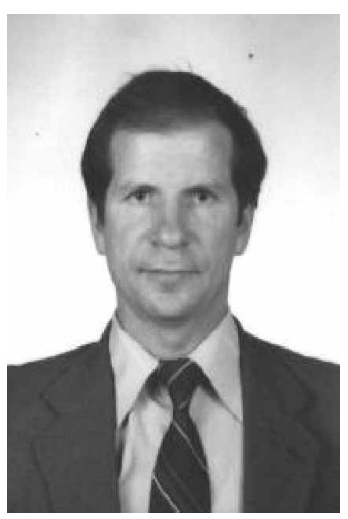

Shutov, Valery graduated from the Electrotechnical Department of Dnipropetrovsky Mining Institute in 1982. His first position was that of an engineer at the sub-faculty of Industrial Process Automation. Since 1991 he was employed as a research officer at the National Mining Academy of Ukraine, Dnipropetrovsk. The areas of his scientific interests cover the design of technological processes for beneficiation of coal and quartz sand and the design of superconducting experimental separators and automatic control systems.

Grebenyuk, Leonid graduated from Dnepropetrovsk State University, and obtained the degree of Candidate of Technical Science (Ph.D) in physics. His scientific interests include mathematical modeling of magnetic separation processes. 